\title{
ASSOCIATION BETWEEN HIGH RISK HUMAN PAPILLOMAVIRUS INFECTION AND SEXUAL TRANSMISSION DISEASES IN WOMEN OF THE METROPOLITAN AREA OF BUCARAMANGA
}

Laura M. Torrado-García ${ }^{1}$, Bladimiro Rincón-Orozco ${ }^{1}$, Ruth Aralí Martínez-Vega ${ }^{1}$, Asunción Olmo ${ }^{2}$

1 Universidad Industrial de Santander, Escuela de Microbiología. Bucaramanga, Colombia ${ }^{2}$ Master Diagnóstica, Granada, Spain

\section{INTRODUCTION}

* High Risk Human Papillomavirus (HR-HPV) has been identified as a necessary cause for the development of cervical cancer (CC) ${ }^{1}$.

* HPV infection is also associated with some factors that include other sexually transmitted diseases (STD). ${ }^{2}$

\section{OBJETIVE}

To evaluate the association between sexually transmitted diseases and HPV infection in women from the metropolitan area of Bucaramanga, Colombia.

\section{METHODS}

Design: Cross-sectional study in women aged 35 to 65 years who were previously screened for HPV because they presented risk factors for developing cervical cancer through an epidemiological survey.

Detection STD: species and serovars of Chlamydia trachomatis, Haemophilus ducreyi, Herpesvirus simplex (HSV-1 / HSV 2), Mycoplasma genitalium, Mycoplasma hominis, Neisseria gonorrhoeae, Treponema pallidum, Trichomonas vaginalis and Ureaplasma (urealyticum / parvum) were detected using multiplex PCR and Specific hybridization (STD Direct Flow CHIP).

Sample: For convenience, 419 samples were collected from September 2016 to November 2018 and prevalence ratios (PR) were measured to find associations using Stata 14 (Figure 1).

Figure 1. Methodological flow chart for detection of sexually transmitted infections

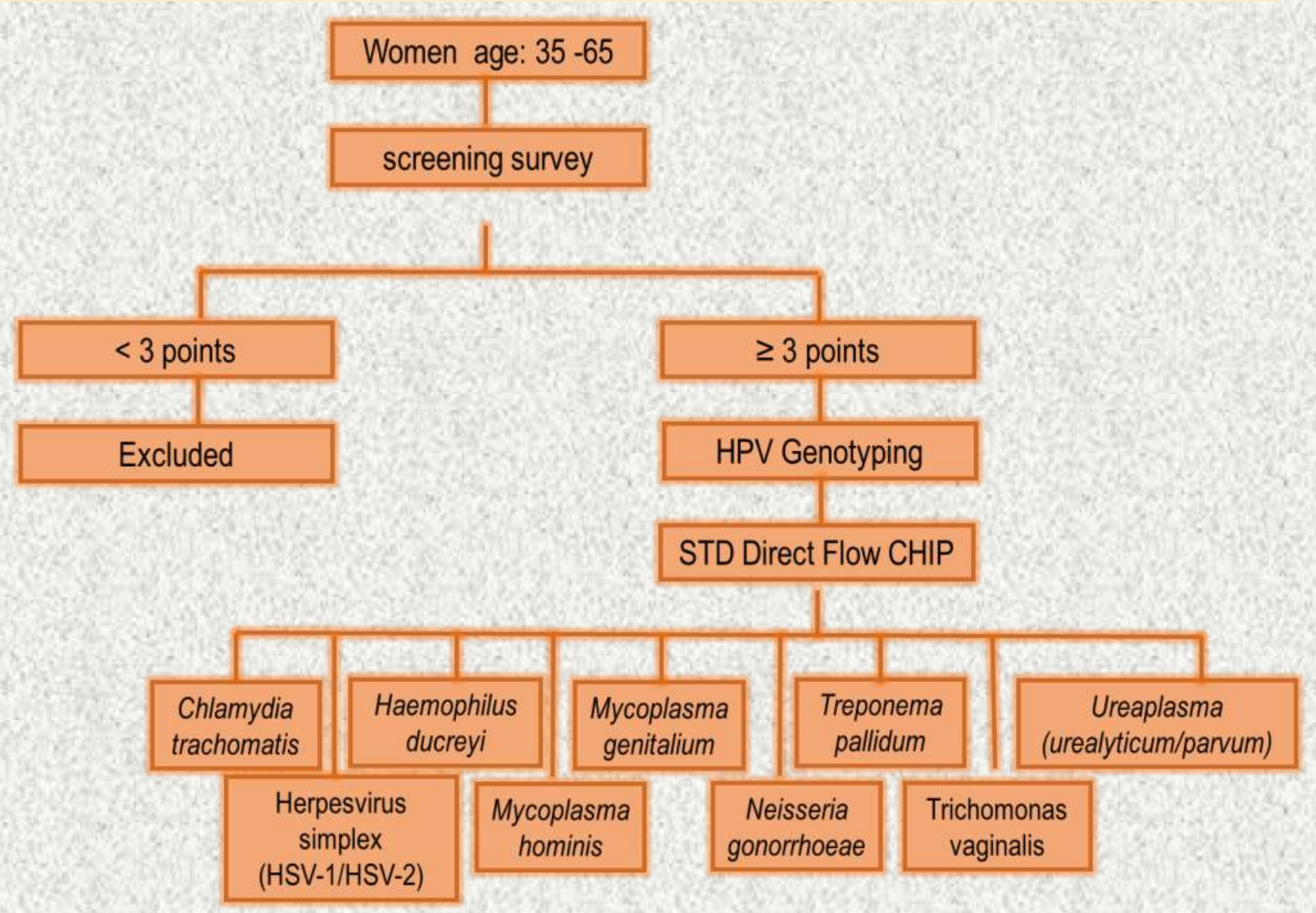

\section{RESULTS AND DISCUSSION}

- The prevalence of HPV-HR with at least one genotype was $27.2 \%(n=114)$.

* The main STD detected in women with HPV-HR infection were Ureaplasma urealyticum/parvum (78\%), Mycoplasma hominis $(43.7 \%)$, Trichomonas vaginalis $(20.3 \%)$, and Herpes simplex virus type II (7.9\%).

* There were association between HPV-HR and STD infections such as: Mycoplasma hominis, Trichomonas vaginalis, Herpes Simplex type 2.
Table 1. Frequency of sexually transmitted infections in patients with and without HPV infection

\begin{tabular}{|c|c|c|c|c|}
\hline Microorganism & $\begin{array}{c}\text { STD frequency HPV } \\
\text { negative } n=305 \\
(72.8 \%)\end{array}$ & $\begin{array}{c}\text { STD frequency HPV } \\
\text { positive } n=114 \\
(27.2 \%)\end{array}$ & $\begin{array}{l}\text { Prevalence ratios } \\
\text { (PR) }\end{array}$ & P value \\
\hline Mycoplasma genitalium & $3(0.24)$ & $3(0.71)$ & - & - \\
\hline Mycoplasma hominis & $107(25.5)$ & $76(18.2)$ & $2.58(1.84-3.62)$ & $<0.0001$ \\
\hline Trichomonas vaginalis & $41(9.6)$ & $45(10.7)$ & $2.56(1.92-3.43)$ & $<0.0001$ \\
\hline $\begin{array}{c}\text { Ureaplasma } \\
\text { urealyticum/parvum }\end{array}$ & $232(55.4)$ & $95(22.7)$ & $1.41(0.91-2.17)$ & 0.12 \\
\hline Haemophilus ducreyi & 0 & 0 & - & - \\
\hline Herpes simplex 1 & $1(0.24)$ & $1(0.24)$ & - & - \\
\hline Herpes simplex 2 & $18(4.3)$ & $15(3.6)$ & $1.77(1.18-2.67)$ & $<0.0001$ \\
\hline Treponema pallidum & 0 & 0 & - & - \\
\hline Neisseria gonorrhoeae & 0 & 0 & - & - \\
\hline $\begin{array}{l}\text { Chlamydia trachomatis } \\
\text { (serovars A-K) }\end{array}$ & $8(1.9)$ & $5(3.1)$ & - & - \\
\hline $\begin{array}{l}\text { Chlamydia trachomatis } \\
\text { (serovars } L 1-L 3 \text { ) }\end{array}$ & $1(0.24)$ & $2(0.48)$ & - & - \\
\hline
\end{tabular}

\section{CONCLUSIONS}

Mycoplasma hominis, Trichomonas vaginalis and Herpes simplex virus type 2 were the most frequent coinfection in HPV-HR + women, and an interesting topic to correlate with cervical cancer development

\section{REFERENCES}

1. Lee SJ, Yang A, Wu TC, Hung CF. Immunotherapy for human papillomavirus-associated disease and cervical cancer: review of clinical and translational research. J Gynecol Oncol. 2016;27:e51.

2. Lukic A, Canzio C, Patella A, Giovagnoli M, Cipriani P, Frega A, et al Determination of cervicovaginal microorganisms in women with abnormal cervical cytology: 